\title{
Lie Symmetry Analysis and Wave Propagation in Variable-Coefficient Nonlinear Physical Phenomena
}

\author{
Mohamed R. Ali ${ }^{1}$, Wen-Xiu Ma ${ }^{2,3,4,5, *}$ and R. Sadat ${ }^{6}$ \\ ${ }^{1}$ Department of Mathematics, Faculty of Engineering, Benha University, Egypt. \\ ${ }^{2}$ Department of Mathematics, Zhejiang Normal University, Jinhua 321004 , \\ Zhejiang, China. \\ ${ }^{3}$ Department of Mathematics, King Abdulaziz University, Jeddah 21589, \\ Saudi Arabia. \\ ${ }^{4}$ Department of Mathematics and Statistics, University of South Florida, \\ Tampa, FL33620-5700, USA. \\ ${ }^{5}$ School of Mathematics, South China University of Technology, Guangzhou \\ 510640, China. \\ ${ }^{6}$ Department of Mathematics, Zagazig Faculty of Engineering, Zagazig University, \\ Zagazig, Egypt.
}

Received 10 September 2020; Accepted (in revised version) 6 January 2021.

\begin{abstract}
We present Lie symmetry analysis to explore solitary wave solutions, twosoliton type solutions and three-soliton type solutions in variable-coefficient nonlinear physical phenomena. An example is a $(2+1)$-dimensional variable-coefficient Bogoyavlensky-Konopelchenko (VCBK) equation. We compute the Lie algebra of infinitesimals of its symmetry vector fields and an optimal system of one-dimensional sub-Lie algebras of the resulting symmetries. Two stages of Lie symmetry reductions will be built to reduce the VCBK equation to nonlinear ordinary differential equations (ODEs) and new analytical solutions to those ODEs will be found by using the integration method. Some of such resulting solutions to the VCBK equation and their dynamics will be illustrated through three-dimensional plots.
\end{abstract}

AMS subject classifications: 76M60, 35Q51, 35C99, 68W30

Key words: Symmetry analysis, partial differential equations, the variable coefficients $(2+1)$-dimensional Bogoyavlensky-Konopelchenko equation.

\section{Introduction}

The $(2+1)$ dimensional Bogoyavlensky-Konopelchenko (BK) equation reads

$$
w_{t}+\alpha w_{x x x}+\beta w_{x x y}+6 \alpha w w_{x}+4 \beta w w_{y}+4 \beta w_{x} \partial_{x}^{-1} w_{y}=0,
$$

$\overline{{ }^{*} \text { Corresponding author. Email address: mawx } @ c a s ~ . u s f ~ . e d u ~(W . X . ~ M a) ~}$ 
where $\alpha$ and $\beta$ are arbitrary constants and $\partial_{x}^{-1}$ is the integral with respect to $x$, cf. $[1,2,16]$. For simplicity, substituting $w(x, y, t)=v_{x}$, we get

$$
v_{x t}+\alpha v_{x x x x}+\beta v_{x x x y}+6 \alpha v_{x} v_{x x}+4 \beta v_{x} v_{x y}+4 \beta v_{y} v_{x x}=0
$$

Many kinds of research $[7,22]$ depicted that $v(x, y, t)$ describes the interaction of a Riemann wave propagating along $y$-axis and $x$-axis. From our fast review, there are many presented solutions for $(1.2)$. Ray $[15,16]$ applied the Lie symmetry analysis to present some generators through the prolongation theorem and the geometric approach, respectively, to reduce (1.2) to ODEs and generate exact solutions. The authors in [9] presented some modifications to Ray's works. Besides, the authors in [23] applied the Lie symmetry method to (1.2) and investigated its conservation laws. Some lump solutions and interacted soliton solutions had been obtained using the Hirota bilinear method in $[5,6,8,11,12,14,18]$. On the other hand, some authors studied the variable-coefficient Bogoyavlensky-Konopelchenko (VCBK) equation

$$
v_{x t}+\alpha(t) v_{x x x x}+\beta(t) v_{x x x y}+\gamma(t) v_{x} v_{x x}+\delta(t) v_{x} v_{x y}+\theta(t) v_{y} v_{x x}=0
$$

where $\alpha(t), \beta(t), \gamma(t), \delta(t)$ and $\theta(t)$ are real functions in time and $\gamma(t)=6 \alpha(t)$ and $\delta(t)=\theta(t)=4 \beta(t)[5,9,13,23,24]$.

The authors of [1] stratified the Hirota method and Bell polynomials to demonstrate the solitons solutions for Eq. (1.3). The interaction between solitons was demonstrated in [13] through utilizing the unified method. Numerous research efforts explore diverse solutions of Eq. (1.3) by various methods such as the inverse scattering method with the aid of Lax pairs and the $G^{\prime} / G$ expansion technique [20-22].

In this paper, we study the VCBK equation, based on the associated Lie algebra [3, 5, 10, 19-23]. Eq. (1.3) has four obscure vectors which were demonstrated in [1], and so we perfect the Lie vectors consisting of arbitrary functions of time through a commutative product for different magnitudes of functions and set the same steps for constant coefficients in Eq. (1.2). Over one or two steps of reduction, several ODEs that have no square are disbanded using the integrating factors as in [17].

In Section 2, we demonstrate three cases for Eq. (1.3). In each case, we use a different value for $\alpha(t), \beta(t), \gamma(t), \delta(t)$ and $\theta(t)$. We examine new solitons and other solutions for the VCBK equation in Section 3 using new Lie vectors that we got in the previous section. In Section 4, we finalise the action by conclusion.

\section{Infinitesimal Generators of Lie Symmetries}

We present three cases, in each one we use a different value of the real function in (1.3). Through the commutative product between the resulted vectors we investigate in each case new infinitesimals for the VCBK equation. 


\subsection{Case I}

Suppose

$$
\alpha(t)=\delta(t)=\frac{t^{2}}{2}, \quad \beta(t)=\frac{t^{2}}{4}, \quad \gamma(t)=\theta(t)=t^{2} .
$$

Eq. (1.3) admits the following Lie symmetry infinitesimal generators:

$$
\begin{aligned}
& X_{1}=f_{1}(t) \frac{\partial}{\partial x}+\frac{\partial}{\partial y}+\left(\frac{1}{t^{2}} y f_{1}^{\prime}(t)+f_{2}(t)\right) \frac{\partial}{\partial v} \\
& X_{2}=f_{3}(t) \frac{\partial}{\partial x}+\frac{1}{t^{2}} \frac{\partial}{\partial t}+\left(\frac{1}{t^{2}} y f_{3}^{\prime}(t)+f_{4}(t)\right) \frac{\partial}{\partial v} \\
& X_{3}=f_{5}(t) \frac{\partial}{\partial x}+t^{3} \frac{\partial}{\partial y}+\left(\frac{1}{t^{2}} y f_{5}^{\prime}(t)+f_{6}(t)+3 x-3 y\right) \frac{\partial}{\partial v}, \\
& X_{4}=\left(x+f_{7}(t)\right) \frac{\partial}{\partial x}+y \frac{\partial}{\partial y}+t \frac{\partial}{\partial t}+\left(\frac{1}{t^{2}} y f_{7}^{\prime}(t)+f_{8}(t)-v\right) \frac{\partial}{\partial v} .
\end{aligned}
$$

There is an infinite number of possibilities for these vectors due to the existence of the arbitrary functions $f_{i}(t), i=1, \ldots, 8$. We derive optimal values for these functions first, by evaluating the commutative product of these infinitesimal generators as listed in Table 1 with

$$
\begin{array}{ll}
a_{1}=\frac{f_{3}^{\prime}}{t^{2}}-\frac{y f_{1}^{\prime \prime}}{t^{3}}+\frac{y f_{1}^{\prime}}{t^{4}}-\frac{f_{2}^{\prime}}{t^{2}}, & a_{2}=3 f_{1}+\frac{1}{t^{2}} f_{5}^{\prime}-3-t^{2} y f_{1}^{\prime}, \\
a_{3}=f_{1}-t f_{1}^{\prime}, & a_{4}=\frac{f_{7}^{\prime}}{t^{2}}-\frac{y f_{1}^{\prime}}{t}-f_{2}-y f_{1}^{\prime \prime}-t f_{2}^{\prime}, \\
a_{5}=3 f_{3}+\frac{y f_{5}^{\prime \prime}}{t^{4}}-2 \frac{y f_{5}^{\prime}}{t^{5}}+\frac{f_{6}^{\prime}}{t^{2}}, & a_{6}=\frac{1}{t^{4}} y f_{7}^{\prime \prime}-\frac{2}{t^{5}} y f_{7}^{\prime}+\frac{f_{8}^{\prime}}{t^{2}}-f_{4}-\frac{1}{t} y f_{3}^{\prime \prime}-t f_{4}^{\prime}, \\
a_{7}=t f_{7}^{\prime}-6 x+6 y-\frac{2 y}{t^{2}} f_{5}^{\prime}-f_{6}-3 f_{7}-\frac{y f_{5}^{\prime \prime}}{t}-2 \frac{y f_{5}^{\prime}}{t^{2}}-t f_{6}^{\prime} .
\end{array}
$$

Simplifying Table 1 by setting the values for $a_{j}$ generates the nonlinear system of ODEs

$$
\begin{array}{lc}
f_{1}^{\prime}=0, \quad f_{7}^{\prime}-2 t^{2} f_{2}-t^{3} f_{2}^{\prime}=0, & f_{5}^{\prime}-3 t^{2} f_{1}=0, \\
3 f_{3}-3 f_{2}-t f_{3}^{\prime}+\frac{1}{t^{2}} f_{6}^{\prime}=0, & -2 f_{3}+\frac{1}{t^{2}} f_{7}^{\prime}-t f_{3}^{\prime}=0, \\
2 f_{4}+t f_{4}^{\prime}-\frac{f_{8}^{\prime}}{t^{2}}=0, & f_{6}+t y f_{7}^{\prime}+\frac{1}{6} t f_{9}^{\prime}-3 f_{7}=0 .
\end{array}
$$

Table 1: Commutator table.

\begin{tabular}{||c|c|c|c|c||}
\hline & $X_{1}$ & $X_{2}$ & $X_{3}$ & $X_{4}$ \\
\hline$X_{1}$ & 0 & $-\frac{f_{1}^{\prime}}{t^{2}} \frac{\partial}{\partial x}+a_{1} \frac{\partial}{\partial v}$ & $a_{2} \frac{\partial}{\partial v}$ & $a_{3} \frac{\partial}{\partial x}+\frac{\partial}{\partial y}+a_{4} \frac{\partial}{\partial v}$ \\
\hline$X_{2}$ & $\frac{f_{1}^{\prime}}{t^{2}} \frac{\partial}{\partial x}-a_{1} \frac{\partial}{\partial v}$ & 0 & $\frac{f_{5}^{\prime}}{t} \frac{\partial}{\partial x}+3 \frac{\partial}{\partial y}+a_{5} \frac{\partial}{\partial v}$ & $\left(f_{3}-t f_{3}^{\prime}\right) \frac{\partial}{\partial x}+\frac{1}{t^{2}} \frac{\partial}{\partial t}+a_{6} \frac{\partial}{\partial v}$ \\
\hline$X_{3}$ & $-a_{2} \frac{\partial}{\partial v}$ & $-\frac{f_{5}^{\prime}}{t} \frac{\partial}{\partial x}-3 \frac{\partial}{\partial y}-a_{5} \frac{\partial}{\partial v}$ & 0 & $\left(f_{5}-t f_{5}^{\prime}\right) \frac{\partial}{\partial x}-2 t^{3} \frac{\partial}{\partial y}+a_{7} \frac{\partial}{\partial v}$ \\
\hline$X_{4}$ & $-a_{3} \frac{\partial}{\partial x}-\frac{\partial}{\partial y}-a_{4} \frac{\partial}{\partial v}$ & $-\left(f_{3}-t f_{3}^{\prime}\right) \frac{\partial}{\partial x}-\frac{1}{t^{2}} \frac{\partial}{\partial t}-a_{6} \frac{\partial}{\partial v}$ & $2 t^{3} \frac{\partial}{\partial y}-\left(f_{5}-t f_{5}^{\prime}\right) \frac{\partial}{\partial x}-a_{7} \frac{\partial}{\partial v}$ & 0 \\
\hline
\end{tabular}


Table 2: Commutator table after optimization.

\begin{tabular}{||c|c|c|c|c||}
\hline & $X_{1}$ & $X_{2}$ & $X_{3}$ & $X_{4}$ \\
\hline$X_{1}$ & 0 & $-\frac{f_{1}^{\prime}}{t^{2}} \frac{\partial}{\partial x}+a_{1} \frac{\partial}{\partial v}$ & $a_{2} \frac{\partial}{\partial v}$ & $X_{1}$ \\
\hline$X_{2}$ & $\frac{f_{1}^{\prime}}{t^{2}} \frac{\partial}{\partial x}-a_{1} \frac{\partial}{\partial v}$ & 0 & $3 X_{1}$ & $3 X_{2}$ \\
\hline$X_{3}$ & $-a_{2} \frac{\partial}{\partial v}$ & $-3 X_{1}$ & 0 & $-2 X_{3}$ \\
\hline$X_{4}$ & $-X_{1}$ & $-3 X_{2}$ & $2 X_{3}$ & 0 \\
\hline
\end{tabular}

Solving this system of differential equations manually and the assumption of some values results in

$$
\begin{aligned}
& f_{1}=c_{1}, \quad f_{5}=c_{1} t^{3}, \quad f_{4}=t^{-4}, \quad f_{7}=\frac{2}{3} c_{2} t^{3}, \\
& f_{3}=c_{2}, \quad f_{6}=0, \quad f_{2}=c_{2} .
\end{aligned}
$$

Substituting (2.4) in (2.1), we explore the four unknown Lie symmetry infinitesimal generators and then simplify Table 1 to an optimized form described in Table 2

$$
\begin{aligned}
& X_{1}=c_{1} \frac{\partial}{\partial x}+\frac{\partial}{\partial y}+c_{2} \frac{\partial}{\partial v}, \\
& X_{2}=c_{2} \frac{\partial}{\partial x}+\frac{1}{t^{2}} \frac{\partial}{\partial t}+t^{-4} \frac{\partial}{\partial v}, \\
& X_{3}=c_{1} t^{3} \frac{\partial}{\partial x}+t^{3} \frac{\partial}{\partial y}+\left(3 c_{1} y+3 x-6 y\right) \frac{\partial}{\partial v}, \\
& X_{4}=\left(x+\frac{2}{3} c_{2} t^{3}\right) \frac{\partial}{\partial x}+y \frac{\partial}{\partial y}+t \frac{\partial}{\partial t}+\left(2 c_{2} y+c_{3}-v\right) \frac{\partial}{\partial v} .
\end{aligned}
$$

\subsection{Case II}

Assume

$$
\alpha(t)=\frac{t}{2}, \quad \beta(t)=\frac{t}{5}, \quad \gamma(t)=t, \quad \delta(t)=\theta(t)=\frac{2 t}{5} .
$$

Eq. (1.3) admits the following Lie symmetry infinitesimal generators:

$$
\begin{aligned}
& X_{1}=f_{1}(t) \frac{\partial}{\partial x}+\frac{\partial}{\partial y}+\left(\frac{5}{2 t} y f_{1}^{\prime}(t)+f_{2}(t)\right) \frac{\partial}{\partial v} \\
& X_{2}=f_{3}(t) \frac{\partial}{\partial x}+\frac{1}{t} \frac{\partial}{\partial t}+\left(\frac{5}{2 t} y f_{3}^{\prime}(t)+f_{4}(t)\right) \frac{\partial}{\partial v} \\
& X_{3}=f_{5}(t) \frac{\partial}{\partial x}+t^{2} \frac{\partial}{\partial y}+\left(\frac{5\left(f_{5}^{\prime}(t)-5 t\right) y}{2 t}+f_{6}(t)+5 x\right) \frac{\partial}{\partial v}, \\
& X_{4}=\left(\frac{2}{3} x+f_{7}(t)\right) \frac{\partial}{\partial x}+\frac{2}{3} y \frac{\partial}{\partial y}+t \frac{\partial}{\partial t}+\left(\frac{5}{2 t} y f_{7}^{\prime}(t)+f_{8}(t)-\frac{2}{3} v\right) \frac{\partial}{\partial v} .
\end{aligned}
$$


Following the same steps as in Case I, we investigate the values of $f_{1}(t), f_{2}(t), f_{3}(t), f_{4}(t)$, $f_{5}(t), f_{6}(t), f_{7}(t)$ and $f_{8}(t)$, i.e.

$$
\begin{aligned}
& f_{1}=c_{1}, \quad f_{5}=c_{1} t^{2}, \quad f_{4}=t^{-8 / 3}, \quad f_{7}=\frac{2}{3} c_{2} t^{2}, \\
& f_{3}=c_{2}, \quad f_{6}=c_{3}, \quad f_{2}=\frac{5}{2} c_{2} .
\end{aligned}
$$

Substituting from (2.7) in (2.6), we examine the unknown Lie symmetry infinitesimal generators as follow:

$$
\begin{aligned}
& X_{1}=c_{1} \frac{\partial}{\partial x}+\frac{\partial}{\partial y}+\frac{5}{2} c_{2} \frac{\partial}{\partial v}, \\
& X_{2}=c_{2} \frac{\partial}{\partial x}+\frac{1}{t} \frac{\partial}{\partial t}+t^{-8 / 3} \frac{\partial}{\partial v}, \\
& X_{3}=c_{1} t^{2} \frac{\partial}{\partial x}+t^{2} \frac{\partial}{\partial y}+\left(5 c_{1} y-\frac{25}{2} y+c_{3}+5 x\right) \frac{\partial}{\partial v}, \\
& X_{4}=\left(\frac{2}{3} x+\frac{2}{3} c_{2} t^{2}\right) \frac{\partial}{\partial x}+\frac{2}{3} y \frac{\partial}{\partial y}+t \frac{\partial}{\partial t}+\left(\frac{10}{3} c_{2} y+c_{3}-\frac{2}{3} v\right) \frac{\partial}{\partial v} .
\end{aligned}
$$

\subsection{Case III}

Assume $\alpha(t)=1, \beta(t)=1$, so, $\gamma(t)=6$ and $\delta(t)=\theta(t)=4$. Eq. (1.3) admits the following Lie symmetry infinitesimal generators:

$$
\begin{aligned}
& X_{1}=f_{1}(t) \frac{\partial}{\partial x}+\frac{\partial}{\partial t}+\left(\frac{1}{4} y f_{1}^{\prime}(t)+f_{2}(t)\right) \frac{\partial}{\partial v}, \\
& X_{2}=f_{3}(t) \frac{\partial}{\partial x}+\frac{\partial}{\partial y}+\left(\frac{1}{4} y f_{3}^{\prime}(t)+f_{4}(t)\right) \frac{\partial}{\partial v}, \\
& X_{3}=f_{5}(t) \frac{\partial}{\partial x}+t \frac{\partial}{\partial y}+\left(\frac{f_{5}^{\prime}(t) y}{4}+f_{6}(t)+\frac{1}{4} x-\frac{3}{8} y\right) \frac{\partial}{\partial v}, \\
& X_{4}=\left(\frac{1}{3} x+f_{7}(t)\right) \frac{\partial}{\partial x}+\frac{1}{3} y \frac{\partial}{\partial y}+t \frac{\partial}{\partial t}+\left(\frac{1}{4} y f_{7}^{\prime}(t)+f_{8}(t)-\frac{1}{3} v\right) \frac{\partial}{\partial v} .
\end{aligned}
$$

Following the same procedure as in the previous cases yields

$$
\begin{aligned}
& f_{3}=c_{1}, \quad f_{1}=8 c_{2} \frac{t}{(3 t-1)}, \quad f_{5}=\frac{8}{3} c_{2} t, \\
& f_{4}=c_{2}, \quad f_{7}=c_{1} t, \quad f_{8}=t^{1 / 3} \\
& f_{2}=c_{3}, \quad f_{6}=-2 y c_{2} \frac{1}{(3 t-1)^{2}}-2 y c_{2} \frac{1}{9(3 t-1)}+\frac{4}{3} c_{3} t .
\end{aligned}
$$

Substituting (2.10) in (2.9), we generate new Lie symmetry infinitesimal generators as in Eq. (2.11), i.e.

$$
X_{1}=\frac{8 c_{2} t}{(3 t-1)} \frac{\partial}{\partial x}+\frac{\partial}{\partial t}+\left(y\left(\frac{2 c_{2}}{(3 t-1)}-\frac{6 c_{2} t}{(3 t-1)^{2}}\right)+c_{3}\right) \frac{\partial}{\partial v},
$$




$$
\begin{aligned}
& X_{2}=c_{1} \frac{\partial}{\partial x}+\frac{\partial}{\partial y}+c_{2} \frac{\partial}{\partial v}, \\
& X_{3}=\frac{8}{3} c_{2} t \frac{\partial}{\partial x}+t \frac{\partial}{\partial y}+\left(\frac{2 c_{2} y}{3}-\frac{2 y c_{2}}{(3 t-1)^{2}}-\frac{2 y c_{2}}{9(3 t-1)}+\frac{4}{3} c_{3} t+\frac{1}{4} x-\frac{3}{8} y\right) \frac{\partial}{\partial v}, \\
& X_{4}=\left(\frac{1}{3} x+c_{1} t\right) \frac{\partial}{\partial x}+\frac{1}{3} y \frac{\partial}{\partial y}+t \frac{\partial}{\partial t}+\left(\frac{c_{1}}{4} y+t^{1 / 3}-\frac{1}{3} v\right) \frac{\partial}{\partial v} .
\end{aligned}
$$

\section{Reduction of Independent Variables in the VCBK Equation}

\subsection{Case I}

Applying the Lie vector $X_{12}=X_{2}-X_{1}$ in Eq. (2.1) gives

$$
X_{12}=X_{2}-X_{1}=-\frac{\partial}{\partial y}+\frac{1}{t^{2}} \frac{\partial}{\partial t}+\left(t^{-4}-1\right) \frac{\partial}{\partial v} .
$$

Hence, the characteristic equation is

$$
\frac{d x}{0}=\frac{d y}{-1}=\frac{d t}{t^{-2}}=\frac{d v}{\left(t^{-4}-1\right)} .
$$

Solving this equation leads to

$$
v(x, y, t)=F(r, s)-\frac{1}{3} t^{3}-\frac{1}{t},
$$

where $F(r, s)$ is the new independent variable and

$$
r=x, \quad s=3 y+t^{3} .
$$

Using the new similarity and the dependent variables, Eq. (1.3) is reduced to

$$
3 F_{r s}+\frac{1}{2} F_{r r r r}+\frac{3}{4} F_{r r r s}+F_{r} F_{r r}+\frac{3}{2} F_{r} F_{r s}+3 F_{s} F_{r r}=0 .
$$

Eq. (3.5) has the analytic solution

$$
F(r, s)=c_{4}-\frac{6 c_{2} \tanh \left(c_{1}+c_{2} r-(2 / 3) c_{2}^{3} s /\left(c_{2}^{2}+1\right)\right)}{2 c_{2}^{2}-1} .
$$

Using the similarity variables in (3.3) and (3.4) then we can carry out the back substitution to the original variables to obtain

$$
v(x, y, t)=c_{4}-\frac{6 c_{2} \tanh \left(c_{1}+c_{2} x-(2 / 3) c_{2}^{3}\left(3 y+t^{3}\right) /\left(c_{2}^{2}+1\right)\right)}{2 c_{2}^{2}-1}-\frac{1}{3} t^{3}-\frac{1}{t} .
$$

This solution is plotted as depicted in Fig. 1. 


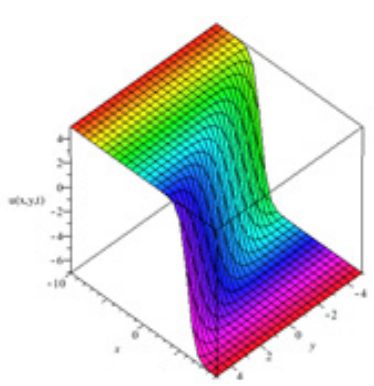

(a)

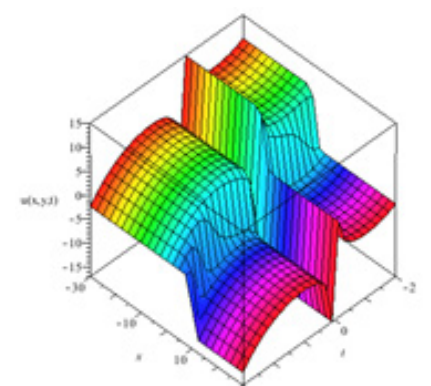

(b)

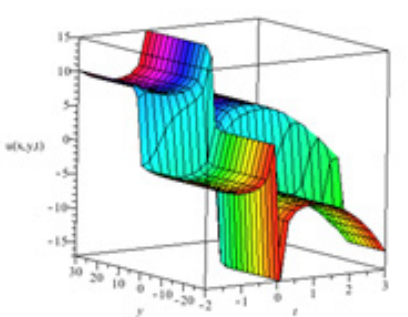

(c)

Figure 1: Three dimensional plots for $u(x, y, t)$ at $c_{1}=-1, c_{2}=1, c_{4}=1$ : (a) $t=0.5$, (b) $y=0$ and (c) $x=0$.

\subsection{Case II}

Applying the Lie vector $X_{1}$ in Eq. (2.6) gives

$$
X_{1}=\frac{\partial}{\partial x}+\frac{\partial}{\partial y}+\frac{5}{2} \frac{\partial}{\partial v} \text {. }
$$

This vector generates the characteristic equation

$$
\frac{d x}{1}=\frac{d y}{1}=\frac{d v}{5 / 2}
$$

Solving (3.9) produces the similarity variables

$$
v(x, y, t)=F(r, s)+\frac{5}{2} x,
$$

where $F(r, s)$ is the new independent variable and

$$
r=-x+y, \quad s=t .
$$

Using the new similarity and the dependent variables, Eq. (1.3) will be reduced to

$$
-F_{r s}+\frac{3}{10} s F_{r r r r}-\frac{1}{5 s} F_{r} F_{r r}+\frac{3}{2} s F_{r r}=0 .
$$

Hence, the Eq. (3.12) has eight Lie vectors, and we choose to work with

$$
V_{13}=\frac{\partial}{\partial r}+\frac{1}{s} \frac{\partial}{\partial s}+2 \frac{\partial}{\partial F}
$$

where

$$
\eta=-2 r+s^{2}, \quad \theta(\eta)=F(r, s)-2 r .
$$

Eq. (3.12) is reduced to

$$
12 \theta_{\eta \eta \eta \eta}+21 \theta_{\eta \eta}+4 \theta_{\eta \eta} \theta_{\eta}=0 .
$$

This equation has no analytical solution, and so we try to solve it using the integrating factor method. 
Integration Procedure. Integrating (3.14) once with respect to $\eta$, we set the integration constant equal to zero,

$$
\theta_{\eta \eta \eta}=-\frac{1}{6} \theta_{\eta}^{2}-\frac{7}{4} \theta_{\eta}
$$

Secondly, multiply (3.14) by $\left(\theta_{\eta}\right)$ and integrate once with respect to $\eta$,

$$
\theta_{\eta \eta \eta}=\frac{1}{72\left(\theta_{\eta}\right)}\left(-8 \theta_{\eta}^{3}+36 \theta_{\eta \eta}^{2}-63 \theta_{\eta}^{2}\right)
$$

Equating Eqs. (3.15) and (3.16) results in

$$
4 \theta_{\eta}^{3}+63 \theta_{\eta}^{2}+36 \theta_{\eta \eta}^{2}=0
$$

by solving this equation

$$
\begin{aligned}
\theta(\eta)= & -9 \sqrt{7} \tan \left(\frac{1}{4} \sqrt{7} \eta+\frac{1}{4} \sqrt{7} c_{1}\right)+9 \sqrt{7} \tan ^{-1}\left(\tan \left(\frac{1}{4} \sqrt{7} \eta+\frac{1}{4} \sqrt{7} c_{1}\right)\right) \\
& -\frac{63}{4} \eta+c_{2} .
\end{aligned}
$$

By back substitution using Eqs. (3.13), (3.11) and (3.10), we get

$$
\begin{aligned}
v(x, y, t)= & -9 \sqrt{7} \tan \left(\frac{1}{4} \sqrt{7}\left(-2(-x+y)+t^{2}\right)+\frac{1}{4} \sqrt{7} c_{1}\right) \\
& +9 \sqrt{7} \tan ^{-1}\left(\tan \left(\frac{1}{4} \sqrt{7}\left(-2(-x+y)+t^{2}\right)+\frac{1}{4} \sqrt{7} c_{1}\right)\right) \\
& -\frac{63}{4}\left(-2(-x+y)+t^{2}\right)+c_{2}+2(-x+y)+\frac{5}{2} x .
\end{aligned}
$$

The result is depicted in Fig. 2. The resulted waves consist of three solitons and while time increases, the amplitude of the wave is reduced and moved to the right.

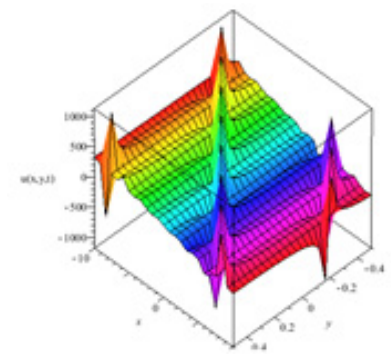

(a)

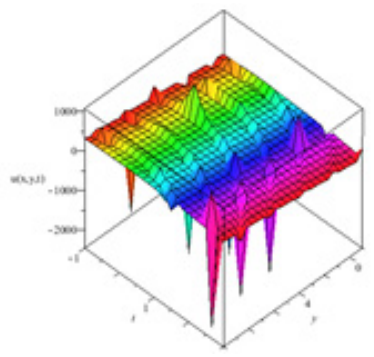

(b)

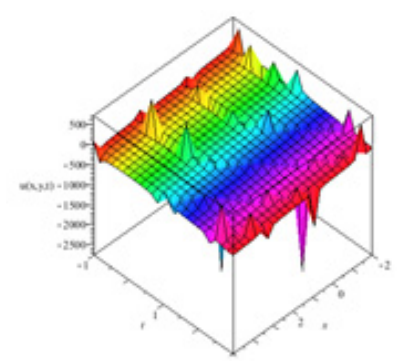

(c)

Figure 2: Three dimensional plots for $u(x, y, t)$ : (a) $t=0$, (b) $x=0$ and (c) $y=0$. 


\subsection{Case III}

Applying the Lie vector $X_{1}-X_{2}$ in (2.11), we have

$$
X_{12}=\left(\frac{8 t}{(3 t-1)}-1\right) \frac{\partial}{\partial x}-\frac{\partial}{\partial y}+\frac{\partial}{\partial t}+\left(y\left(\frac{2 c_{2}}{(3 t-1)}-\frac{6 c_{2} t}{(3 t-1)^{2}}\right)\right) \frac{\partial}{\partial v} .
$$

So, the characteristic equation will be

$$
\frac{d x}{(8 t /(3 t-1)-1)}=\frac{d y}{-1}=\frac{d t}{1}=\frac{d v}{\left(y\left(2 c_{2} /(3 t-1)-6 c_{2} t /(3 t-1)^{2}\right)\right)}
$$

Solving this equation leads to

$$
v(x, y, t)=F(r, s)+\frac{4 c_{2}}{3(3 t-1)}+\frac{1}{4} x+\frac{13}{36}-\frac{5}{12} t,
$$

where $F(r, s)$ is a new independent variable and

$$
r=-x+\frac{8}{9} \ln (3 t-1)+\frac{5}{3} t, \quad s=-\frac{1}{3}+t+y .
$$

Using the new similarity and the dependent variables, Eq. (1.3) is reduced to

$$
-\frac{1}{6} F_{r r}-2 F_{r s}+F_{r r r r}-F_{r r r s}+4 F_{s} F_{r r}+4 F_{r} F_{r s}-6 F_{r} F_{r r}=0 .
$$

Eq. (3.24) has the analytic solution

$$
F(r, s)=c_{4}+\frac{39}{4} \frac{c_{2} \tanh \left(c_{1}+c_{2} r+\left(c_{2}\left(24 c_{2}^{2}-1\right) s\right) /\left(12\left(2 c_{2}^{2}+1\right)\right)\right)}{3 c_{2}^{2}-1} .
$$

Using the similarity variables in (3.22) and (3.23), we can do the back substitution to the original variables to obtain

$$
v(x, y, t)=c_{4}+\frac{39}{4} \frac{U(t, y)}{3 c_{2}^{2}-1}+\frac{4 c_{2}}{3(3 t-1)}+\frac{1}{4} x+\frac{13}{36}-\frac{5}{12} t
$$

where

$$
U(t, y)=c_{2} \tanh \left(c_{1}+c_{2}\left(-x+\frac{8}{9} \ln (3 t-1)+\frac{5}{3} t\right)+\frac{c_{2}\left(24 c_{2}^{2}-1\right)(-1 / 3+t+y)}{12\left(2 c_{2}^{2}+1\right)}\right) .
$$

The result is plotted in Fig. 3. 


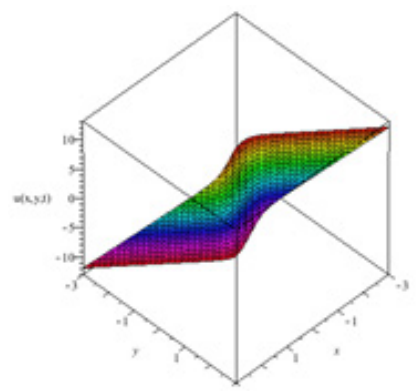

(a)

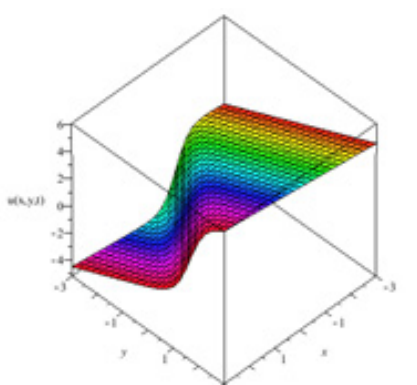

(b)

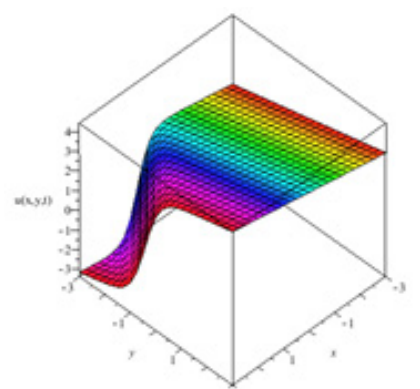

(c)

Figure 3: Three dimensional plots for $u(x, y, t)$ for $c_{1}=1, c_{2}=2, c_{4}=-1 / 18$ : (a) $t=0.4$, (b) $t=0.6$ and (c) $t=1$.

\section{Conclusions}

We studied a $(2+1)$-dimensional variable-coefficient Bogoyavlensky-Konopelchenko (VCBK) equation. We showed that the (2+1)-dimensional VCBK equation admits an infinite number of Lie point symmetries. We determined an optimal system of one-dimensional subalgebras of the Lie point symmetries. Abundant exact travelling wave solutions, two-soliton type solutions and three-soliton type solutions were presented. The summary of our results is as follows:

1. The inspected Lie vectors are distinct and fairly similar to $[1,15]$.

2. In view of the new Lie vectors, we minimized Eq. (1.3) to several ODEs and resolved those conditions utilizing explicit integration techniques.

3. We can examine our layouts as follows:

(I) Figs. 1(a), 2(a), 3(a-c) suggest that one-soliton and three-soliton solutions rely upon the expansion in time esteem. The amplitude is decreasing with expanding time and the wave tops move to a lift bearing.

(II) Figs. 1(b,c) address the collaboration among the multi-soliton waves and this implies the actual properties do not change after the interaction.

(III) Figs. 2(b,c) explore periodical and parabolical waves.

\section{References}

[1] M.A. Abdulwahhab, Comment on the paper "On conservation laws by Lie symmetry analysis for (2+1)-dimensional Bogoyavlensky-Konopelchenko equation in wave propagation" by S. Saha Ray, Comput. Math. Appl. 75, 4300-4304 (2018).

[2] M.R. Ali, A Truncation Method for Solving the Time-Fractional Benjamin-Ono Equation, J. Appl. Math. 2019, 1v-7 (2019).

[3] M.R. Ali and W.X. Ma, New exact solutions of Bratu Gelfand model in two dimensions using Lie symmetry analysis, Chinese J. Phys. 65, 198-206 (2020). 
[4] G.Baumann, Symmetry Analysis of Differential Equations with Mathematica ${ }^{\circledR}$, Springer Science \& Business Media (2013).

[5] S.T. Chen and W.X. Ma, Lump solutions to a generalized Bogoyavlensky-Konopelchenko equation, Front. Math. China 13(3), 525-534 (2018).

[6] S.T. Chen and W.X. Ma, Exact solutions to a generalized Bogoyavlensky-Konopelchenko equation via maple symbolic computations, Complexity 2019, (2019).

[7] A.K. Halder, A. Paliathanasis and P. Leach, Similarity solutions and conservation laws for the Bogoyavlensky-Konopelchenko equation by Lie point symmetries, ArXiv 2003, 10131 (2020).

[8] V. Jadaun and S. Kumar, Symmetry analysis and invariant solutions of $(3+1)$-dimensional Kadomtsev-Petviashvili equation, Int. J. Geom. Methods Mod. Phys. 1850125 (2018).

[9] Q. Li, T. Chaolu and Y.H. Wang, Lump-type solutions and lump solutions for the (2+1)-dimensional generalized Bogoyavlensky-Konopelchenko equation, Comput. Math. with Appl. 77, 2077-2085 (2019).

[10] W.X. Ma, M.R. Ali and R. Sadat, Analytical solutions for nonlinear dispersive physical model, Complexity 2020, Article D 3714832 (2020).

[11] W.X. Ma, Z. Qin and X. Lü, Lump solutions to dimensionally reduced p-gKP and p-gBKP equations, Nonlinear Dyn. 84, 923-931 (2016).

[12] J. Manafian, B.I. Mohammadi and M. Abapour, Breather wave, periodic, and cross-kink solutions to the generalized Bogoyavlensky-Konopelchenko equation, Math. Methods Appl. Sci. 43, 1753-1774 (2020).

[13] M. Osman and J. Machado, The dynamical behavior of mixed-type soliton solutions described by (2+1)-dimensional Bogoyavlensky-Konopelchenko equation with variable coefficients, J. Electromagn. Waves Appl. 32(11), 1457-1464 (2018).

[14] C. Qin, S. Tian and T. Zhang, Lie symmetry analysis, conservation laws and exact solutions of fourth-order time fractional Burgers equation, J. Appl. Anal. Comput. 8(6), 1727-1746 (2018).

[15] S.S. Ray, On conservation laws by Lie symmetry analysis for (2+1)-dimensional BogoyavlenskyKonopelchenko equation in wave propagation, Comput. Math. Appl. 74, 1158-1165 (2017).

[16] S.S. Ray, Lie symmetry analysis and reduction for exact solution of (2+1)-dimensional Bogoyavlensky-Konopelchenko equation by geometric approach, Modern Phys. Lett. B 32(11), 1850127 (2018).

[17] R. Sadat, M. Kassem and W.X. Ma, Families of analytic solutions for $(2+1)$ model in unbounded domain via optimal Lie vectors with integrating factors, Modern Phys. Lett. B 33, 1950229 (2019).

[18] S.F. Tian, Lie symmetry analysis, conservation laws and solitary wave solutions to a fourth-order nonlinear generalized Boussinesq water wave equation, Appl. Math. Lett. 100, 106056 (2020).

[19] H. Triki, Z. Jovanoski and A. Biswas, Shock wave solutions to the Bogoyavlensky-Konopelchenko equation, Indian J. Phys. 88, 71-74 (2014).

[20] D.S. Wang, and Y. Yin, Symmetry analysis and reductions of the two-dimensional generalized Benney system via geometric approach, Computers \& Mathematics with Applications 71, 748757 (2016).

[21] D.S. Wang and H.Q. Zhang, Further improved F-expansion method and new exact solutions of Konopelchenko-Dubrovsky equation, Chaos, Solitons \& Fractals. 25, 601-610 (2005).

[22] M. Wang, X. Li and J. Zhang, The $\left(G^{\prime} G\right)$-expansion method and travelling wave solutions of nonlinear evolution equations in mathematical physics, Phys. Lett. A 372(4), 417-423 (2008).

[23] Y.L. Wang, Y.T. Gao, S.L. Jia, G.-F. Deng and W.Q. Hu, Solitons for a (2+1)-dimensional variablecoefficient Bogoyavlensky-Konopelchenko equation in a fluid, Modern Phys. Lett. B 31(25), 1750216 (2017).

[24] H. Yan, S.F. Tian, L.L. Feng and T.T. Zhang, Quasi-periodic wave solutions, soliton solutions, 
and integrability to a (2+1)-dimensional generalized Bogoyavlensky-Konopelchenko equation, Waves Random Complex Media 26(4), 444-457 (2016).

[25] T.T. Zhang, On Lie symmetry analysis, conservation laws and solitary waves to a longitudinal wave motion equation, Appl. Math. Lett. 98, 199-205 (2019). 